\title{
Long-Term Outcomes After Stress Urinary Incontinence Surgery
}

\author{
Michele Jonsson Funk, PhD, Nazema Y. Siddiqui, MD, MHSc, mie Kawasaki, MD, and \\ Jennifer M. Wu, MD, MPH \\ Department of Epidemiology, Gillings School of Global Public Health, and the Center for \\ Women's Health Research, University of North Carolina at Chapel Hill, Chapel Hill, and the \\ Department of Obstetrics and Gynecology, Duke University, Durham, North Carolina
}

\section{Abstract}

OBJECTIVES-To compare the long-term risk of repeat stress urinary incontinence (SUI) surgery after different types of initial SUI surgery and to identify predictors of time to repeat SUI surgery in a large, population-based cohort.

METHODS-We used de-identified, adjudicated health care claims data from approximately 100 employer-based plans across the United States from 2000 to 2009. We identified the index SUI surgery in women aged 18 to 64 years. Kaplan-Meier survival curves were used to estimate cumulative incidence of repeat surgery through 9 years. Cox proportional hazards models were used to estimate the adjusted hazard ratios (HRs) and 95\% confidence intervals (CIs) for factors associated with recurrent SUI surgery.

RESULTS-Over 10 years, we identified 155,458 eligible women who underwent one or more SUI surgeries, with a total of 294,855 person-years of follow-up. Of these index surgeries, $127,848(82.2 \%)$ were slings. The 9-year cumulative incidence of repeat surgery after any SUI surgery was $14.5 \%$ (95\% CI 13.4-15.5). As expected, bulking agents had the highest cumulative incidence of repeat surgery $(61.2 \%, 95 \%$ CI $56.3-66.0)$ followed by needle suspension $(22.2 \%$, 95\% CI 16.5-27.9); the lowest 9-year incidences were for Burch (10.8\%, 95\% CI 9.3-12.3) and sling (13.0\%, 95\% CI 11.7-14.3). In a Cox proportional hazards model that adjusted for age, year of index surgery, and region of the United States, the rate of repeat surgery was $28 \%$ higher for slings compared with Burch (adjusted HR 1.28, 95\% CI 1.19-1.37).

CONCLUSION-In this population-based analysis of women aged 18 to 64 years, Burch procedures had the lowest 9-year cumulative incidence of repeat SUI surgery.

Surgical management of stress urinary incontinence (SUI) is common in the United States with approximately 95,000 inpatient procedures ${ }^{1}$ and 105,500 outpatient procedures per year. ${ }^{2}$ Numerous surgical procedures exist for the treatment of SUI. Historically, the gold standard was the Burch colposuspension which was first introduced in 1961 and involves supporting the anterior vaginal wall to Cooper ligament through a laparotomy incision. ${ }^{3,4}$ The midurethral mesh sling has now supplanted the Burch as the new standard. This shift has occurred fairly rapidly given that the first midurethral sling, the tension-free vaginal tape

(C) 2012 by The American College of Obstetricians and Gynecologists. Published by Lippincott Williams \& Wilkins.

Corresponding author: Dr. Jennifer M. Wu, Duke University Medical Center, Box 3192, 5324 McFarland Avenue, Suite 310, Durham, NC 27707; jennifer.wu@duke.edu.

Presented at the 38th annual meeting of the Society of Gynecologic Surgeons, April 13-15, 2012, Baltimore, Maryland.

The content is solely the responsibility of the authors and does not necessarily represent the official views of the Agency for Healthcare Research and Quality.

Financial Disclosure

The authors did not report any potential conflicts of interest. 
(TVT), was approved by the U.S. Food and Drug Administration (FDA) just 14 years ago in 1998.5

One important factor that led to the wide adoption of the TVT was a randomized trial in which 344 women with SUI were treated with either TVT or Burch and found to have comparable long-term cure and complication rates. ${ }^{6,7}$ Furthermore, the minimally invasive midurethral sling has the advantages of shorter operative times, technical ease, and the ability to be performed on an outpatient basis, all of which have added to the popularity of this procedure.

Existing literature suggests that the overall risk of reoperation after an initial SUI surgery is approximately $8 \%-9 \%{ }^{8,9}$ However, when estimating the risk of repeat SUI surgery based on the type of initial surgery, there have been conflicting results. Fialkow et al found that the sling had a higher rate of reoperation compared with the Burch colposuspension. ${ }^{8}$ In contrast, Abdel-Fattah et al reported higher rates of repeat SUI surgery after Burch. ${ }^{9}$ Thus, it is unclear which procedure is associated with higher rates of repeat SUI surgery. Even fewer data are available regarding the risk of repeat surgery after other types of SUI procedures. Furthermore, limited data exist regarding differences in the risk of reopera-tion for SUI based on age, region of the United States, or the year in which the initial surgery was performed.

Given the limitations in the existing literature, the primary objective of this study is to use a large health care claims database to estimate the long-term rate of reoperation for SUI after an initial SUI surgery. Our secondary objective was to identify predictors of time to repeat SUI surgery in a large, population-based cohort.

\section{MATERIALS AND METHODS}

We used the Thomson Reuters MarketScan Commercial Claims and Encounters database (health care claims database) from 2000 to $2009 .{ }^{10}$ This database contains de-identified, individual-specific clinical utilization and enrollment claims data for inpatient and outpatient visits from approximately 100 employer-based plans in the United States. These deidentified data represent the medical experience of privately insured employees, their spouses, and their dependents until the age of 65, when they become eligible for Medicare. This database has been rigorously evaluated and is valid and reliable. ${ }^{11}$ Furthermore, the weighted data are representative of the younger, non-Medicare, privately insured U.S. population in terms of sex, age, and region, ${ }^{12}$ and unique individuals can be tracked longitudinally using encrypted identification numbers. This database provides information on approximately 3.7 million individuals from 2000 and increases to 40.0 million individuals by 2009. This study was determined to be exempt from further review by the Institutional Review Board at the University of North Carolina at Chapel Hill.

The population at risk included all women aged 18 to 64 years from 2000 to 2009. All SUI procedures among eligible women were identified based on the following Physicians' Current Procedural Terminology Coding System, $4^{\text {th }}$ edition (CPT) codes: 57288 for sling; 51840, 51841, or 51851 for Burch colposuspension; 51715 for endoscopic injection of implant material into the submucosal tissue of the urethra, which we will refer to as "bulking agents"; 57220 for Kelly plication; 51845 or 57289 for needle suspension; 51990 or 51992 for laparoscopic urethral suspension or laparoscopic sling operation, respectively, which is referred to as "laparoscopic"; and 58267 or 58293 for total vaginal hysterectomy with colpourethrocystopexy.

After capturing all SUI procedures, the first, or index, SUI surgery for each woman was identified. We included women with at least 90 days of prior enrollment who did not have 
any gaps in coverage. Those with a diagnosis of a urethral diverticulum (International Classification of Diseases, $9^{\text {th }}$ Revision code 619.0) or any urinary- genital tract fistula (International Classification of Diseases, $9^{\text {th }}$ Revision codes 599.1 and 599.2) in the 90 days before the index surgery were excluded. If both an inpatient and outpatient procedure claim occurred on the same service date, we preferentially included the inpatient procedure. In addition, if two or more procedure codes were observed on the same index date of surgery, the primary procedure for the index SUI surgery was assigned using a hierarchy which generally reflects decreasing degree of invasiveness: 1) Burch followed by 2) laparoscopic SUI procedure, 3) total vaginal hysterectomy with colpo-urethrocystopexy, 4) sling, 5) needle suspension, 6) bulking agent, and 7) Kelly plication.

To evaluate the long-term outcomes after SUI surgery, we estimated the cumulative incidence of repeat SUI surgery stratified by the type of index surgery. Repeat SUI surgery was defined as any subsequent SUI surgery. In this analysis, we did not include sling revision or removal (CPT 57287) as we were focused on repeat surgery for the treatment of SUI. We estimated the cumulative incidence and 95\% confidence intervals (CIs) of repeat SUI surgery annually through 9 years of follow-up for each type of index surgery. We also assessed the following predictors for repeat surgery: age at the time of the index procedure (18-34, 35- 44, 45-54, and 55- 64 years), geographic region (Northeast, Midwest, South, West), and calendar year at the time of index procedure (2000-2009).

Descriptive statistics were used to characterize the population of women undergoing index SUI surgery. Kaplan-Meier survival curves were used to estimate the cumulative incidence of repeat SUI surgery annually for each year of follow-up, stratified by type of index surgery. For the Kaplan-Meier analysis, a log-rank test was performed to assess differences among the groups. We used Cox proportional hazards models to estimate the adjusted hazard ratio (HR) of repeat surgery for each decade of increasing age at the time of the index surgery compared with those 18 to 34 years, for calendar year at the time of the index surgery relative to 2000, for region of the United States compared with the Northeast, and for each type of procedure compared with the Burch. To further examine the changes over time in the risk of repeat surgery for sling compared with Burch, we estimated separate hazard ratios for each year of surgery. We also evaluated for possible interaction between age and sling relative to Burch. We estimated $95 \%$ CIs on all effect estimates. Statistical analyses were performed using SAS 9.2.

\section{RESULTS}

In this national database of privately insured individuals from 2000 to 2009, there were a total of 32.9 million women aged 18 to 64 years. Of these women, 168,844 underwent one or more SUI procedures, and for each of these women, we identified their first SUI surgery. Among 155,458 women who met our inclusion criteria, the median age of women who underwent an index SUI surgery was 49 years (interquartile range 43-56, range 18-64). Table 1 depicts the SUI surgery type-specific details regarding age, region, and calendar year in which the surgery was performed. Patients who had a Burch were nearly 2 years older, on average, than those who had a sling (median age 47.1 compared with 49.0 years). The median year of the index procedure was 2007 (interquartile range 2005-2008). The median length of follow-up after the index procedure was 1.3 years (interquartile range $0.5-$ 2.7 years, range $0-9.75$ years), which represented a total of 294,855 person-years. The median length of follow-up was not longer because the database includes many more individuals in recent years and, thus, the majority of SUI surgeries included in the analysis occurred during those years. For example, 3.7 million individuals were in the database in 2000 compared with 40.0 million in 2009 . Thus, the opportunity to follow up women before administrative censoring on December 31,2009, was shortened and this decreased the 
overall median length of follow-up. The cumulative incidence of repeat SUI surgery for all types of surgery at 9 years of follow-up was $14.5 \%$ (95\% CI 13.4-15.5). The type of repeat surgery performed was sling $(70.5 \%)$ followed by bulking agents $(20.1 \%)$, Burch $(6.5 \%)$, laparoscopic $(1.5 \%)$, needle $(0.8 \%)$, total vaginal hysterectomy $(0.5 \%)$, and Kelly $(0.2 \%)$. Table 2 depicts the rate of repeat surgery for each year after the index surgery, stratified by type of index procedure. As expected, bulking agents had the highest incidence of repeat surgery followed by needle suspension; the Burch procedure had the lowest incidence, which was $10.8 \%$ (95\% CI 9.3-12.3) at 9 years of follow-up. Kaplan-Meier survival curves depict the cumulative proportion of patients who underwent repeat SUI surgery over time and accounts for the fact that not all patients were followed for the full 9 years (Fig. 1). The Kaplan-Meier survival analysis demonstrates that there is a statistically significant difference among the groups $(P<.001)$. Because the rate of repeat surgery for bulking agents was so high, we also estimated the incidence of repeat surgery after excluding this procedure. With bulking agents excluded, the cumulative incidence of repeat SUI surgery remained unchanged at $14.5 \%$ (95\% CI 13.4-15.5), which reflects the fact that bulking agents represented only a small fraction of the total number of surgeries.

To assess predictors of repeat surgery, we performed a Cox proportional hazards model and evaluated age, calendar year when the index surgery was performed, region of the United States, and type of index SUI procedure (Table 3). Women aged 35 to 44 years and 45 to 54 years did not have a significant difference in rate of repeat surgery compared with women aged 18 to 34 years. However, women aged 55 to 64 years had a 14\% higher rate of repeat surgery compared with women aged 18 to 34 years (adjusted HR 1.14, 95\% CI 1.04-1.25). Compared with women who had the index surgery in 2000 , the rate of repeat surgery was significantly lower in all subsequent years (2001-2009). When compared with the Northeast region of the United States, the rate of repeat surgery was elevated in the South (adjusted HR 1.66, 95\% CI 1.52-1.82) and in the West (adjusted HR 1.24, 95\% CI 1.12-1.38) but was not significantly different in the Midwest (adjusted HR 1.06, 95\% CI 0.96-1.17).

After adjusting for age, calendar year of index surgery, and region, the rate of repeat surgery was 8-fold higher for bulking agents (adjusted HR 8.19, 95\% CI 7.53-8.90) compared with Burch. For slings, the rate of repeat surgery was $28 \%$ higher than the rate for Burch procedures (adjusted HR 1.28, 95\% CI 1.19-1.37). Needle suspensions and laparoscopic SUI surgeries also had significantly higher rates of repeat surgery compared with the Burch, whereas the Kelly plication and total vaginal hysterectomy plus colpo-urethrocystopexy were not significantly different (Table 3). Notably, the total number of Kelly plications was quite low (less than $0.3 \%$ of the total procedures), and therefore, we were limited in our ability to detect a statistically significant difference between this procedure and the Burch.

The CPT code for slings (57288) does not distinguish between a midurethral sling compared with traditional bladder neck sling. Because a bladder neck sling is less likely to be performed on an outpatient basis, we conducted a subgroup analysis limited to outpatient slings to better assess the outcomes of midurethral slings in particular. Overall, $66.4 \%$ of slings were performed on an outpatient basis. In an adjusted Cox proportional hazards model, the rate of repeat surgery remained elevated when we compared outpatient slings to the Burch (both inpatient and outpatient) (adjusted HR 1.50, 95\% CI 1.40-1.61).

Because midurethral slings were relatively recently FDA-approved in $1998^{5}$ and have become the most common procedure performed, we wanted to focus on the relative rate of repeat surgery between the sling and the Burch, the previous gold standard. In the adjusted Cox proportional hazards model, the rate of repeat surgery was higher, on average, for slings compared with Burch (Table 3). However, this estimate assumes that any difference in longterm outcomes is the same regardless of the calendar year in which the procedure was 
performed. In other words, this model includes the main effects for calendar year and type of procedure, but does not allow for an interaction between calendar year and type of procedure. In a sensitivity analysis, we included the necessary interaction terms to allow the hazard ratio to vary by age and calendar year. None of the interaction terms for age were statistically significant $(P>.05)$. For calendar year, Figure 2 depicts the adjusted HR for sling compared with Burch, separately for each calendar year at the time of the index surgery. In 2000 and 2001, the hazard rate was lower for sling than Burch; however, between 2002 and 2009 , the hazard rate was significantly higher for sling than Burch, meaning that the rate of repeat surgery was significantly higher for women who had a sling in any year from 2002 to 2009 compared with women who had a Burch in that same time period.

\section{DISCUSSION}

In this population-based analysis of women aged 18 to 64 years, the cumulative incidence of repeat SUI surgery after 9 years of follow-up was $14.5 \%$. We confirmed that less effective SUI surgeries, such as bulking agents ${ }^{13}$ and needle suspension, ${ }^{14}$ have higher rates of repeat surgery. The Burch colposuspension had the lowest rate of repeat surgery for stress incontinence. Of note, the rate of repeat SUI surgery after sling was $28 \%$ higher when compared with the Burch procedure, and this difference was not explained by differences in age or region of the United States. Furthermore, this difference was apparent every year from 2002 to 2009 , and thus, it is unlikely to be related to the initial learning curve for the midurethral sling, which was FDA-approved in $1998 .^{5}$

Both Burch and sling have been shown to be effective in the treatment of $\mathrm{SUI}^{7,15,16}$ but prior observational studies have reported conflicting results regarding whether the Burch or sling has a higher rate of repeat surgery. An analysis of 762 women in the United Kingdom found an overall reoperation rate of $8.8 \%$ after SUI surgery. In this cohort, the risk of repeat SUI surgery was $3.2 \%$ (11/342) for midurethral slings and 10.7\% (34/319) for abdominal retropubic procedures. ${ }^{9}$ In contrast, Fialkow et al evaluated over 41,000 women from Washington state and found that women who received a sling had a higher reoperation rate than those who underwent a Burch (6.7 compared with 4.2 per 1,000 person-years). ${ }^{8}$ Our findings are similar to those of Fialkow et al. Furthermore, our study evaluates a larger number of procedures $(\mathrm{N}=155,458)$ than previous studies and provides long-term, population-based data regarding the risk of reoperation after an initial SUI surgery.

In our analysis, we were able to explore some factors that affect the risk of repeat surgery. Our data are consistent with other studies demonstrating regional variations in rates of surgical procedures for urinary incontinence. In particular, multiple studies have shown lower rates of surgery for urinary incontinence in the Northeast. ${ }^{1,17} \mathrm{We}$ also found that older age was a risk factor for repeat surgery. Clark et al analyzed factors associated with reoperation for pro-lapse and urinary incontinence; the authors did not find age to be associated with repeat surgery among 36 out of 384 women who underwent reoperation. ${ }^{18}$ However, Richter et al found that women 65 years and older were more likely to undergo surgical retreatment for SUI compared with younger women. ${ }^{19}$

The strengths of this study are related to the robust information in the database that we used. Because this database represents a large population-based sample of privately insured women across the United States, information from this database may be more generalizable than data from a single, or even multiple, institutions. In addition, we were able to longitudinally follow patients to assess the rate of repeat SUI surgery and to describe the risk of reoperation based on type of the initial SUI procedure. Because we were able to account for the specific time that each woman was enrolled in the database and to estimate individual 
person-time contribution, we were able to use survival time methods which allow us to identify subsequent surgery for up to 9 years.

Although this database is reliable and valid, there are also several limitations of this data source. Although we were strict in how we defined an index SUI surgery, we do not know if these women had SUI surgery before enrollment into a contributing health plan. Because this database is representative of younger, privately insured individuals, we cannot comment on the relative rate of repeat surgery in elderly women aged 65 and older. This is an important population as SUI surgery is more commonly performed in older women when compared with women less than 65 years of age. ${ }^{1}$ We were also not able to report on other potential risk factors for repeat surgery, such as race or ethnicity or body mass index, as these characteristics are not captured in the database. In addition, we do not have information regarding the severity of symptoms or what type of incontinence existed before the index surgery, for example whether these women had pure stress incontinence compared with mixed incontinence. There may also be other perioperative factors that influenced the choice for regarding the type of index surgery performed and these factors may affect the risk of repeat SUI surgery.

Another limitation is that we were unable to distinguish between midurethral mesh slings compared with traditional bladder neck or pubovaginal slings, given that the CPT code 57288 does not indicate the type of sling performed. Furthermore, among the midurethral slings, we cannot distinguish between the different types such as retropubic, transobturator, or "mini-slings." Thus, we cannot definitively state which type of sling is associated with a higher risk of repeat SUI surgery. However, we know anecdotally that midurethral slings are the predominant surgery for SUI. We attempted to explore this question by conducting a subanalysis of outpatient slings, based on the premise that a bladder neck sling would be less likely to be performed on an outpatient basis. When we focused on outpatient slings, the Burch colposuspension continued to have a lower rate of repeat surgery.

In conclusion, we report that the cumulative rate of repeat SUI surgery was $14.5 \%$ (95\% CI 13.4-15.5) at 9 years of follow-up and that the Burch colposus-pension had the lowest rate of reoperation for SUI. Although we were able to explore age and region of the United States as predictors for the rate of repeat SUI surgery, further research is needed to evaluate other factors that may explain differences in the rate of repeat SUI surgery between the Burch and sling. It is possible that differences in surgical skill among providers may explain the lower rate of repeat surgery for the Burch. Alternatively, owing to the minimally invasive nature of sling surgery, it is possible that surgeons may use less stringent criteria to decide who should undergo a sling. In addition, surgeons may perform a sling in women with mixed incontinence or urge incontinence or in women with other predisposing factors for recurrent urinary incontinence. Given the magnitude of SUI surgeries performed in the United States, it is critically important to evaluate the long-term outcomes of these procedures and to specifically compare different types of SUI surgeries. Based on our data, the Burch procedure remains an effective surgery for SUI with the lowest risk of reoperation for stress incontinence in women aged 18 to 64 years.

\section{Acknowledgments}

Supported by grant K02HS017950 from the Agency for Healthcare Research and Quality (AHRQ). Dr. Wu is supported by K23HD068404, Eunice Kennedy Shriver National Institute of Child Health \& Human Development. The authors thank Rebekah G. Fulton for assistance with formatting the figures and tables. 


\section{References}

1. Wu JM, Gandhi MP, Shah AD, Shah JY, Fulton RG, Weidner AC. Trends in inpatient urinary incontinence surgery in the USA, 1998-2007. Int Urogynecol J. 2011; 22:1437-43. [PubMed: 21975533]

2. Erekson EA, Lopes VV, Raker CA, Sung VW. Ambulatory procedures for female pelvic floor disorders in the United States. Am J Obstet Gynecol. 2010; 203:497. [PubMed: 20739015]

3. Burch JC. Urethrovaginal fixation to Cooper's ligament for correction of stress incontinence, cystocele, and prolapse. Am J Obstet Gynecol. 1961; 81:281-90. [PubMed: 13688914]

4. Lapitan MC, Cody JD, Grant A. Open retropubic colposuspension for urinary incontinence in women. The Cochrane Database of Systematic Reviews. 2009; (4):Art. No.: CD002912.10.1002/14651858.CD002912.pub4

5. Food and Drug Administration. 510(K) Number K974098. Jan 28. 1998 Available at: http:// www.accessdata.fda.gov/scripts/cdrh/cfdocs/cfPMN/pmn.cfm?ID=123614Retrieved November 14, 2011

6. Ward K, Hilton P. Prospective multicentre randomised trial of tension-free vaginal tape and colposuspension as primary treatment for stress incontinence. BMJ. 2002; 325:67. [PubMed: 12114234]

7. Ward KL, Hilton P. Tension-free vaginal tape versus colposuspension for primary urodynamic stress incontinence: 5-year follow up. BJOG. 2008; 115:226-33. [PubMed: 17970791]

8. Fialkow M, Symons RG, Flum D. Reoperation for urinary incontinence. Am J Obstet Gynecol. 2008; 199:546. [PubMed: 18639207]

9. Abdel-Fattah M, Familusi A, Fielding S, Ford J, Bhattacharya S. Primary and repeat surgical treatment for female pelvic organ prolapse and incontinence in parous women in the UK: a register linkage study. BMJ Open. 2011; 1:e000206.

10. Thomson Reuters MarketScan Commercial Claims and Encounters Database, 2000-2009. Available at: http://thomsonreuters.com/products_services/healthcare/healthcare_products/ pharmaceuticals/mktscan_res_db/ Retrieved December 28, 2011

11. Hansen, LG.; Chang, S. Health research data for the real world: the Thomson Reuters MarketScan Databases. White paper. 2011. Available at: http://thomsonreuters.com/content/healthcare/pdf/ white_papers/marketscan_white_paperRetrieve February 16, 2012

12. Pickens, G.; Moldwin, E.; Marder, WD. Healthcare spending index for private insurance: methodology and baseline results (white paper). Nov. 2010 Available at: http:// healthcare.thomsonreuters.com/indexes/assets/HPAYEMP10119145HSI-PIWP_web.pdf. REtrieved December 28, 2011

13. Keegan PE, Atiemo K, Cody J, McClinton S, Pickard R. Periurethral injection therapy for urinary incontinence in women. The Cochrane Database of Systematic Reviews. 2007; 3:Art. No.: CD003881.10.1002/14651858.CD003881.pub2

14. Glazener CM, Cooper K. Bladder neck needle suspension for urinary incontinence in women. The Cochrane Database of Systematic Reviews. 2004; (2):Art. No.: CD003636.10.1002/14651858.CD003636.pub2

15. Albo ME, Richter HE, Brubaker L, Norton P, Kraus SR, Zimmern PE, et al. Burch colposuspension versus fascial sling to reduce urinary stress incontinence. N Engl J Med. 2007; 356:2143-55. [PubMed: 17517855]

16. Park AJ, Barber MD, Bent AE, Dooley YT, Dancz C, Sutkin G, et al. Assessment of intraoperative judgment during gyneco-logic surgery using the Script Concordance Test. Am J Obstet Gynecol. 2010; 203:240. [PubMed: 20494330]

17. Oliphant SS, Wang L, Bunker CH, Lowder JL. Trends in stress urinary incontinence inpatient procedures in the United States, 1979-2004. Am J Obstet Gynecol. 2009; 200:521. [PubMed: 19375571]

18. Clark AL, Gregory T, Smith VJ, Edwards R. Epidemiologic evaluation of reoperation for surgically treated pelvic organ prolapse and urinary incontinence. Am J Obstet Gynecol. 2003; 189:1261-7. [PubMed: 14634551] 
19. Richter HE, Goode PS, Brubaker L, Zyczynski H, Stoddard AM, Dandreo KJ, et al. Two-year outcomes after surgery for stress urinary incontinence in older compared with younger women. Obstet Gynecol. 2008; 112:621-9. [PubMed: 18757661] 


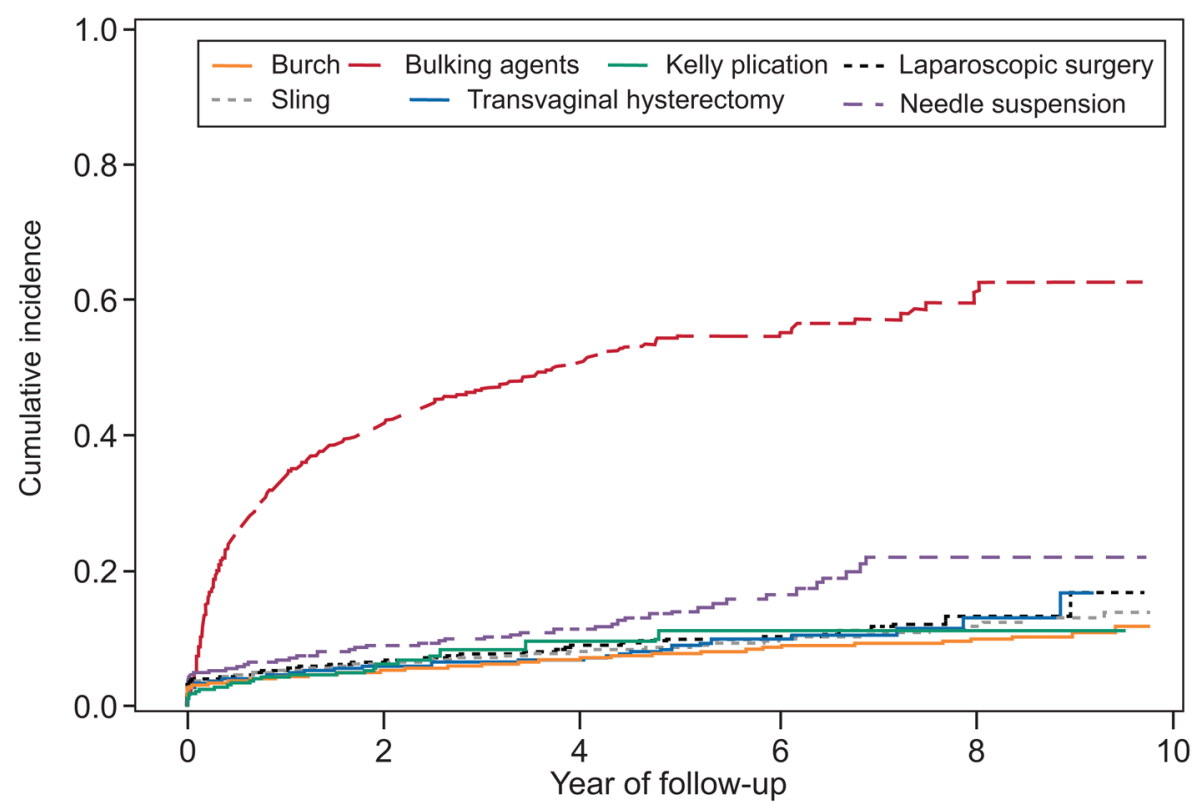

\begin{tabular}{|l|r|r|r|r|r|}
\hline N at risk & Year 0 & Year 2 & Year 4 & Year 6 & Year 8 \\
\hline Burch & 16,670 & 7,906 & 3,875 & 1,849 & 537 \\
\hline Sling & 127,848 & 40,279 & 12,114 & 3,209 & 507 \\
\hline Bulking agents & 4,159 & 879 & 267 & 116 & 28 \\
\hline Laparoscopic surgery & 3,754 & 1,441 & 619 & 267 & 71 \\
\hline Transvaginal hysterectomy & 1,473 & 649 & 336 & 165 & 49 \\
\hline Needle suspension & 1,110 & 485 & 226 & 117 & 34 \\
\hline Kelly plication & 444 & 151 & 64 & 28 & 8 \\
\hline
\end{tabular}

Fig. 1.

Kaplan-Meier survival curve after index stress urinary incontinence (SUI) surgery. This survival curve depicts the cumulative incidence of repeat SUI surgery after each type of procedure, and there is a statistically significant difference among the groups $(P<.001)$. The table lists the at-risk number remaining at the beginning (year 0 ) as well as at years 2, 4, 6, and 8 . 


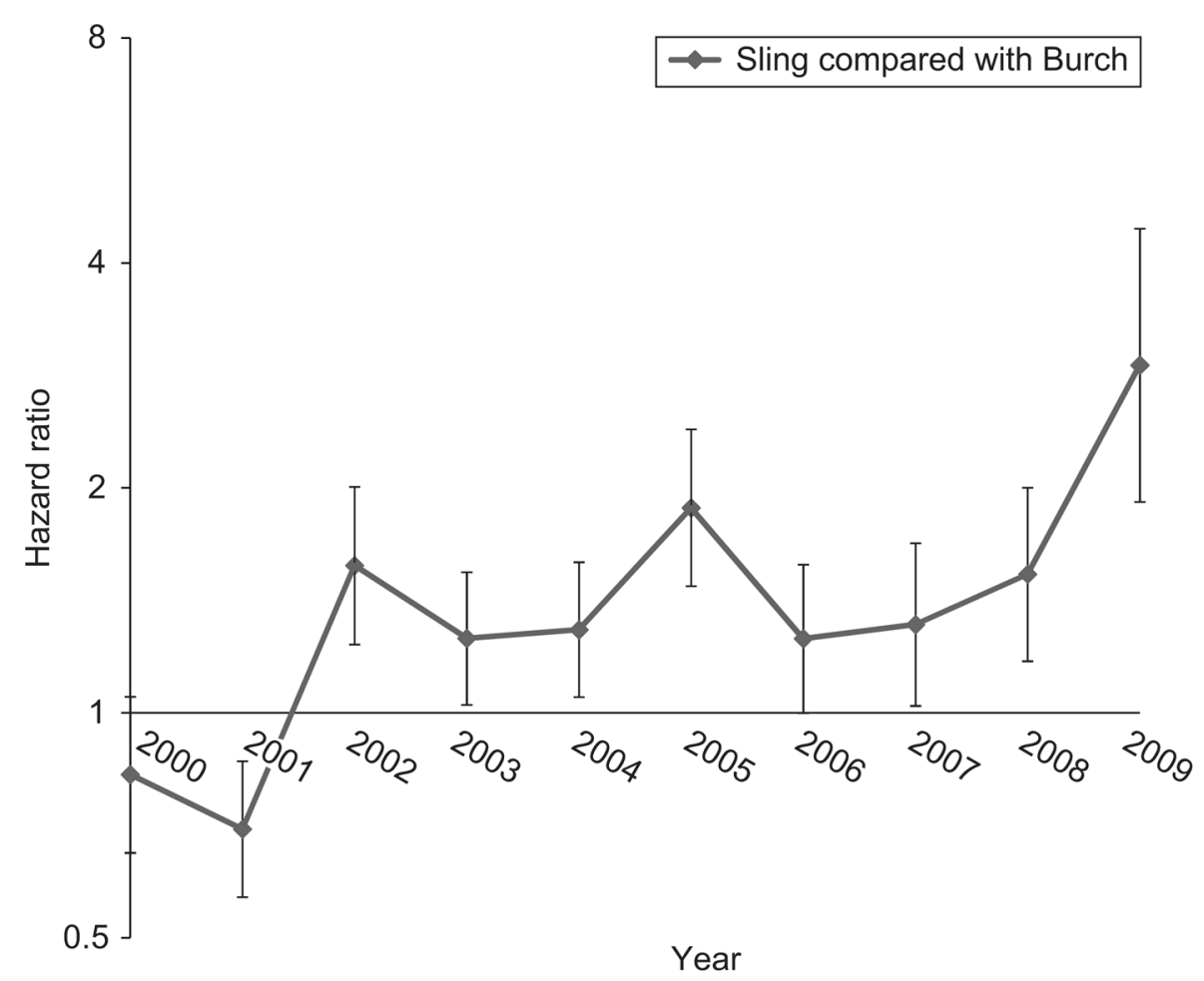

Fig. 2.

The hazard ratio and confidence intervals of repeat surgery after sling compared with Burch for each calendar year. The $\mathrm{Y}$ axis uses a logarithmic scale. 


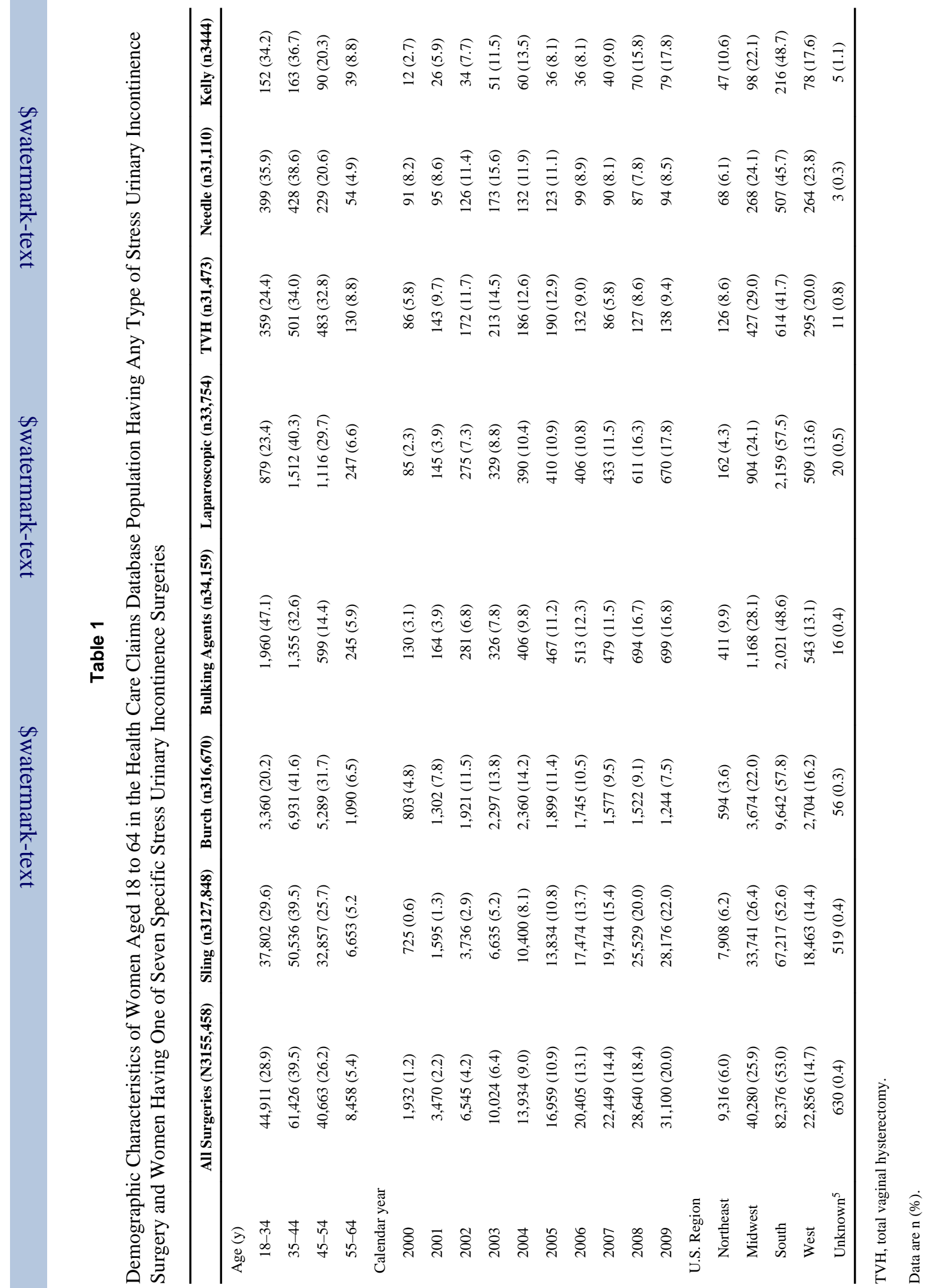




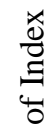

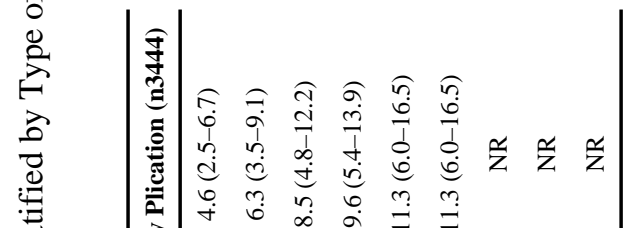

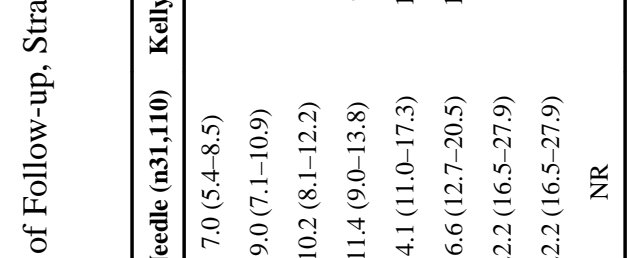

चँ

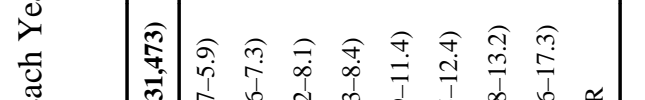

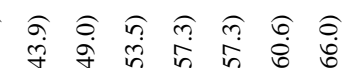

诺 
Table 3

Cox Proportional Hazards Regression Analysis for Time to Repeat Stress Urinary Incontinence Surgery

\begin{tabular}{|c|c|}
\hline Variable & Adjusted Hazard Ratio (95\% Confidence Interval) for Repeat Surgery \\
\hline \multicolumn{2}{|l|}{ Age category $(\mathrm{y})$} \\
\hline 18-34 (reference) & - \\
\hline $35-44$ & $0.95(0.86-1.04)$ \\
\hline $45-54$ & $1.02(0.93-1.12)$ \\
\hline $55-64$ & $1.14(1.04-1.25)$ \\
\hline \multicolumn{2}{|l|}{ Calendar year } \\
\hline 2000 (reference) & - \\
\hline 2001 & $0.71(0.62-0.81)$ \\
\hline 2002 & $0.42(0.37-0.48)$ \\
\hline 2003 & $0.43(0.38-0.49)$ \\
\hline 2004 & $0.42(0.37-0.47)$ \\
\hline 2005 & $0.50(0.45-0.57)$ \\
\hline 2006 & $0.43(0.38-0.48)$ \\
\hline 2007 & $0.41(0.37-0.47)$ \\
\hline 2008 & $0.49(0.44-0.55)$ \\
\hline 2009 & $0.56(0.49-0.62)$ \\
\hline \multicolumn{2}{|l|}{ Region of the United States } \\
\hline Northeast (reference) & - \\
\hline Midwest & $1.06(0.96-1.17)$ \\
\hline South & $1.66(1.52-1.82)$ \\
\hline West & $1.24(1.12-1.38)$ \\
\hline Unknown & $1.73(1.29-2.31)$ \\
\hline \multicolumn{2}{|l|}{ SUI surgery type } \\
\hline Burch (reference) & - \\
\hline Sling & $1.28(1.19-1.37)$ \\
\hline Bulking agents & $8.19(7.53-8.90)$ \\
\hline Laparoscopic & $1.29(1.12-1.48)$ \\
\hline TVH and colpo-urethrocystopexy & $1.18(0.95-1.45)$ \\
\hline Needle suspension & $1.75(1.43-2.13)$ \\
\hline Kelly plication & $1.15(0.78-1.70)$ \\
\hline
\end{tabular}

SUI, stress urinary incontinence; TVH, total vaginal hysterectomy. 